\title{
Frequency of asymptomatic choroidal metastasis in patients with disseminated breast cancer: results of a prospective screening programme
}

\begin{abstract}
Thomas Wiegel, Klaus Martin Kreusel, Norbert Bornfeld, Dirk Bottke, Margot Stange,
\end{abstract} Michael H Foerster, Wolfgang Hinkelbein

\begin{abstract}
Aim-To determine the frequency of visually asymptomatic choroidal metastasis in patients with disseminated breast cancer and its dependence on the incidence of metastasis by number and site of other organ metastases.

Methods-From January 1995 until April 1997120 patients irradiated for disseminated breast cancer underwent ophthalmological screening for choroidal metastasis. No patient was symptomatic for ocular disease. 68 out of 120 patients were found to have metastases in one organ and 52 patients had metastases in more than one organ. $80 \%$ of the patients had bone metastases, $25 \%$ lung metastases, $22 \%$ liver metastases, $15 \%$ brain metastases, and $22 \%$ had metastases in other organs.

Results-Six patients (5\%) were found to have asymptomatic choroidal metastases. Five patients had unilateral and one patient bilateral metastases. 52 patients with more than one involved organ had a significantly higher risk for asymptomatic choroidal metastasis $(6 / 52,11 \%)$ than 68 patients with metastases in only one organ $(0 / 68)(p=0.006)$. In univariate analysis a significantly higher risk was seen for patients with lung metastases (14\% choroidal metastases versus $2 \%$ in patients without lung metastases, $p=0.03$ ) and for patients with brain metastases $(17 \%$ choroidal metastases versus $3 \%$ in those without brain metastases, $p=0.04$ ). Conclusion-In disseminated breast cancer the incidence of asymptomatic choroidal metastases was $5 \%$ and increased to $11 \%$ when more than one organ was involved in metastatic spread. Risk factors for choroidal metastases were dissemination of disease in more than one organ and the presence of lung and brain metastases. (Br f Ophthalmol 1998;82:1159-1161)
\end{abstract}

Intraocular metastasis is the most common intraocular malignancy. ${ }^{1}$ In up to $80-90 \%$ of all female patients breast cancer is the primary tumour. ${ }^{2-4}$ The overall survival of these patients is poor with a median of 8-12 months for all patients and 15-17 months for patients with breast cancer. ${ }^{5}$ However, if choroidal metastasis is detected early enough, it can be treated effectively with radiation therapy, hormonal therapy, and/or chemotherapy in order to prevent loss of vision and thus maintain quality of life. ${ }^{356}$

Some research groups have evaluated the incidence of ocular metastases. ${ }^{7} \mathrm{~A}$ few series of necropsies examined the eye status, ${ }^{8-11}$ but only two studies looked for the incidence of these metastases in patients dying from disseminated breast cancer. ${ }^{911}$

Only two other clinical studies investigated the rate of choroidal metastases in living patients with disseminated breast cancer but
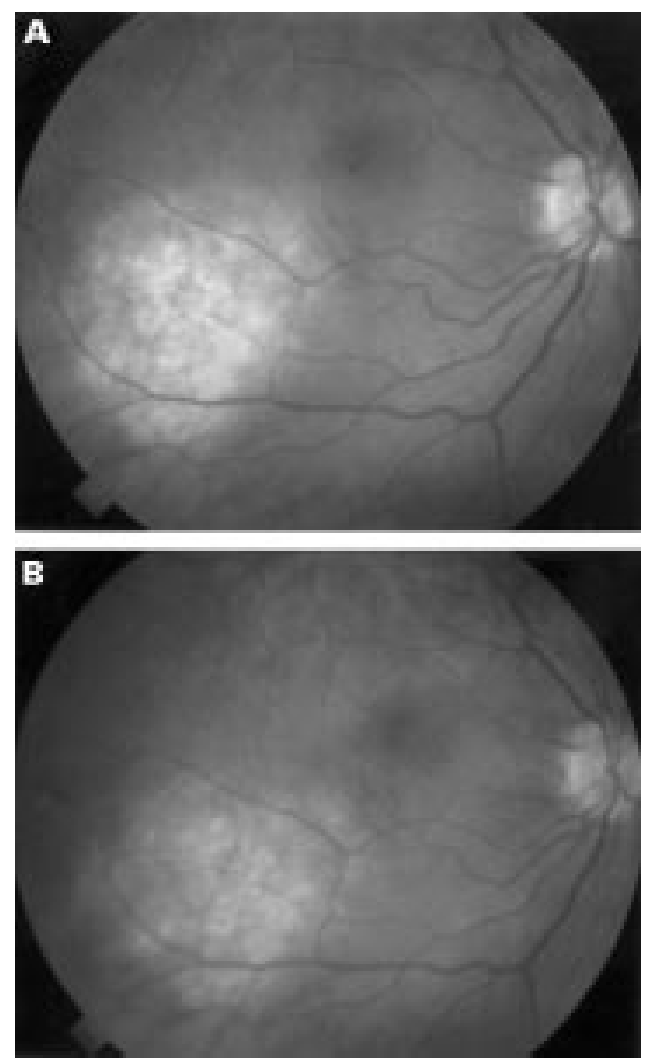

Figure 1 (A) Asymptomatic metastasis extending into the macula. Visual acuity was 20/20, sonographic prominence was $1.9 \mathrm{~mm}$. (B) After external beam irradiation the metastasis showed complete regression and visual function could be preserved (follow up time 33 months).

Metastases in other than the four organ systems-for example, skin, were stated as "others". For every patient all sites of metastases were stated. 

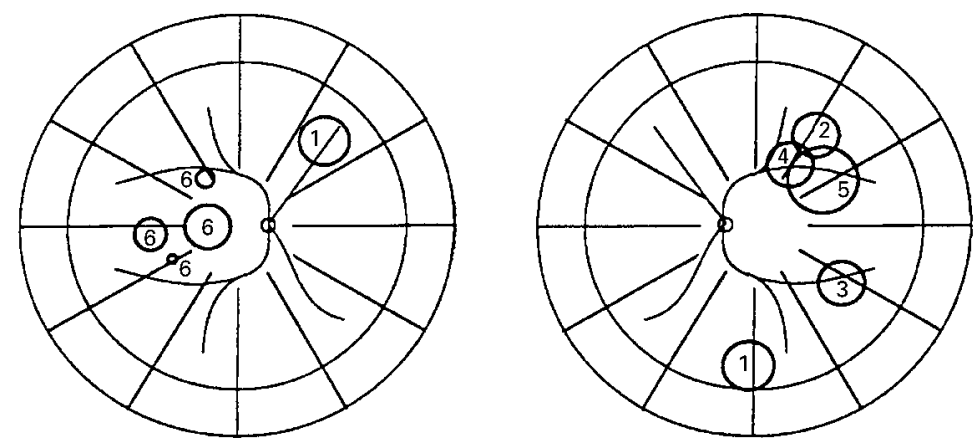

Figure 2 Localisation and approximate extension of choroidal metastases detected. None of the metastases was symptomatic. Numbers indicate numbers of patients; in one patient metastases were found in both eyes.

without ocular symptoms and found an incidence of between $7 \%$ and $9 \% .{ }^{8}{ }^{12}$ However, both groups stated only that all of their patients had disseminated breast cancer and no detailed information was given about the stage of metastatic disease.

To determine the frequency of choroidal metastases in patients with disseminated breast cancer and the dependence on the number and extraocular site of metastases, a clinical screening programme was conducted at the University Hospital Benjamin Franklin in 1995.

\section{Material and methods}

The study consisted of patients with histologically proved disseminated breast cancer (M1), treated with radiation therapy for symptomatic metastases at the Department of Radiation Oncology of the University Hospital Benjamin Franklin. Between 1995 and 1997, 120 patients with a median age of 55 years (range 34-89 years) were referred to the department of ophthalmology for ophthalmological screening for choroidal metastasis. The patients were unselected and represented the majority of all patients with disseminated breast cancer treated at this institution. None of these patients was symptomatic for ocular disease. Patients with symptomatic choroidal metastases were excluded from this study. Detailed information on the primary tumour and the stage of metastatic disease is given in Table 1. All patients were screened for metastases of the following organs: brain, liver, lung, and bones. Metastases were identified by computed tomograph scans or by ultrasound of the brain, chest, and abdomen and for bone metastases by technetium scintigraphy. Metastases in other organ systems were stated as "others". If available, the primary tumour stage ( $\mathrm{T} 1 / 2$ or

Table 2 Analysis of factors influencing the incidence of choroidal metastasis in disseminated breast cancer

\begin{tabular}{lll}
\hline & $\begin{array}{l}\text { Univariate } \\
\text { (p value) }\end{array}$ & $\begin{array}{l}\text { Relative risk } \\
(95 \% \text { CI) }\end{array}$ \\
\hline Age & 0.95 & \\
Time from primary diagnosis & 0.70 & \\
Primary stage T1/2 versus T3/4 & 0.64 & \\
Primary node status: positive versus node negative & 1.00 & not possible \\
Primary receptor status: positive versus negative & 0.34 & $1.32(0.2-10.8)$ \\
One versus more than one site of metastasis & 0.006 & $6.6(1.3-34)$ \\
Bone metastasis & 0.60 & $5.7(1.2-26)$ \\
Lung metastasis & 0.03 & $0.7(0.1-6)$ \\
Brain metastasis & 0.04 & \\
Liver metastasis & 0.62 & \\
\hline
\end{tabular}

Table 3 Results of data pooling of all three studies

\begin{tabular}{|c|c|c|}
\hline & Mamma-Ca & $(95 \% C I)$ \\
\hline (1) Philadelphia ${ }^{1}$ & $4 / 52(9.2 \%)$ & $2.1-18.5 \%$ \\
\hline (2) Mayo Clinic ${ }^{15}$ & $9 / 98(9.7 \%)$ & $4.3-16.7 \%$ \\
\hline (3) Berlin, UKBF & $6 / 120(5 \%)$ & $1.8-10.6 \%$ \\
\hline (Sum 1-3) & $19 / 270(7 \%)$ & $4.6-11.6 \%$ \\
\hline
\end{tabular}

T3/4), the primary lymph node status (positive or negative), and the receptor status (positive or negative) of the breast cancer was documented. Data were not available in $13 \%$ for the primary stage (16 out of 120 ), in $12 \%$ for the primary lymph node status (15 out of 120), and in $6 \%$ for the receptor status (seven out of 120) owing to the long history of their disease.

All patients underwent ophthalmological screening which comprised visual acuity, slit lamp examination, and binocular ophthalmoscopy. Choroidal metastases were identified by their typical ophthalmoscopic aspect, displaying a greyish white appearance with mottling of the pigment epithelium and visible prominence (Fig 1). The prominence of the lesions was confirmed by A and B-scan ultrasonography.

The incidence of metastases was correlated with the number of organs involved in metastatic spread and the site of metastases. The results were compared statistically using Fisher's exact test. ${ }^{13}$ Differences were considered to be significant when $\mathrm{p}<0.05$ was detected.

\section{Results}

The median time from diagnosis of the primary breast cancer to choroidal screening of all patients was 43 months (range 0-286 months). Altogether, six out of 120 (5\%, 95\% confidence interval (CI) $1.8-10.6 \%$ ) patients had choroidal metastases. Five patients had unilateral and one patient bilateral metastases. Localisation and approximate size of metastases are displayed in Figure 2.

Choroidal metastases were only found in patients with two or more other sites of systemic metastases. No patient with only one site of metastasis had choroidal metastasis (0/68, 95\% CI $0 \%-6 \%$ ) compared with $6 / 52$ patients with more than one site of metastasis $(6 / 52,95 \% \mathrm{CI}$ $4 \%-23 \%)(p=0.006)$. The incidence of choroidal metastasis in the latter group was $11 \%$.

A significantly higher risk was seen for patients with lung and brain metastases; four out of 28 patients with lung metastases (and possible more metastases) had choroidal metastasis but only two out of 92 patients without lung metastases $(\mathrm{p}=0.03$, relative risk $6.6-95 \%$ CI 1.3-34)). Three out of 18 patients with brain metastases had choroidal metastases but only three out of 102 without brain metastases had choroidal metastases ( $\mathrm{p}=0.04$, relative risk $5.7-95 \%$ CI 1.2-26). This was not seen for patients with liver metastases (1/26 v 5/94, $\mathrm{p}=0.62$, relative risk 0.72 ) or bone metastases (5/95 $v 1 / 25, \mathrm{p}=0.60$, relative risk 1.32) (Table $2)$. The primary tumour stage (T1/2 versus $T$ $3 / 4$ ), the lymph node status (node positive versus node negative) and the receptor status (receptor positive versus receptor negative) had no significant influence on the rate of choroidal metastases. 


\section{Discussion}

The aim of our study was to determine the incidence of asymptomatic choroidal metastases in patients with disseminated breast cancer and to evaluate the correlation between the incidence of choroidal metastasis and the stage of metastatic disease. This is of great interest for oncologists as there are treatment options (radiation therapy, chemotherapy, hormonal treatment) which are highly effective to improve visual acuity in symptomatic disease and which help to maintain the quality of the patient's life. ${ }^{614-18}$

Taking into consideration that the rate of ocular metastasis is even higher in postmortem studies since occult microscopic disease can be detected, $4-10 \%$ of all patients dying of disseminated cancer had ocular metastases. Nelson et al ${ }^{11}$ observed 15 patients with ocular metastases $(4 \%)$ in 376 patients dying of all types of carcinoma. Eliassi-Rad et al ${ }^{9}$ demonstrated 25 cases of ocular metastases $(5.1 \%)$ in 510 patients dying of carcinomas at the Wilmer Eye Institute, Baltimore. The incidence of these metastases was $8.3 \%(3 / 36)$ and $9.7 \%$ (3/31) for patients dying with disseminated breast cancer. ${ }^{911}$ In 515 patients dying between 1950 and 1965 with disseminated cancer five microscopic choroidal metastases were found, thus giving an incidence of only $0.9 \% .{ }^{10}$

In 1967 Albert et al ${ }^{1}$ stated that in four out of 52 patients $(7.7 \%)$ choroidal metastases were detected. This was in good agreement with Mewis and Young, who found nine patients with choroidal metastases in 98 patients $(9.2 \%)$ without ocular symptoms. ${ }^{12}$ However, all study populations concerning this question were small and, more importantly, the site and number of systemic metastases were not stated.

From retrospective analyses it is well known that the median time from diagnosis of cancer to the onset of ocular symptoms is about $40-60$ months for all patients and about 6-10 months for patients with lung cancer. ${ }^{2419}$ The mean time of follow up in our 97 patients was 54 months and the median time 43 months and is exactly within the range where choroidal metastases can be expected.

Necropsy studies have shown that up to $80 \%$ of patients with symptomatic choroidal metastasis also have lung metastases. ${ }^{57}$ This is in good agreement with our finding that patients with lung metastases have a significantly higher risk of developing choroidal metastasis. Thus, patients with breast cancer and lung metastases are at a relatively high risk of developing choroidal metastasis and could profit from early diagnosis.

Owing to the wide confidence intervals of all three studies (including ours) it seems neces- sary to pool these data; the study designs were comparable and the methods of detecting choroidal metastases nearly the same. Then, the incidence of choroidal metastasis in disseminated breast cancer patients is $7 \%(19 /$ 270 ) and the $95 \%$ CI varies from $4.6 \%$ to $11.6 \%$ (Table 3 ). In our study this incidence increased up to $11 \%$ in patients with more than one site of metastases. Patients with lung and brain metastases have a significantly higher risk of choroidal metastasis compared with patients with only one site of metastasis or without lung or brain involvement.

In the present study choroidal metastasis could be demonstrated to be a frequent finding in disseminated breast cancer. Since we were able to identify risk factors for this vision threatening complication of disease, ophthalmological screening could be considered in high risk patients.

1 Albert DM, Rubenstein RA, Scheie HG. Tumor metastasis to the eye. Part I: Incidence in 213 adult patients with generalized malignancy. Am f Ophthalmol 1963; 63:723-6.

2 Shields CL, Shields JA, Gross NE, et al. Survey of 520 eyes with uveal metastases. Ophthalmology 1997;104:1265-76.

3 Wiegel T, Kleineidam M, Schilling A. Choroidal metastasis in a patient with adenocarcinoma of the cervix. Strahlenther Onkol 1995;171:539-42.

4 Wiegel T, Bornfeld N, Kreusel KM, et al. Radiotherapy for choroidal metastases interim analysis of a prospective study of the ARO (ARO 95-08). Front Radiat Ther Oncol 1997;30:154-9.

5 Debois JM, Haustrate FM. Breast cancer and the eye. Onkologie 1996;19:385-93.

6 Letson AD, Davidorf FH, Bruce RA. Chemotherapy for treatment of choroidal metastases from breast carcinoma. Am F Ophthalmol 1982;93:102-6.

7 Weiss L. Analysis of the incidence of intraocular metastasis. Br f Ophthalmol 1993;77:149-51.

8 Bloch RS, Gartner S. The incidence of ocular metastatic carcinoma. Arch Ophthalmol 1971;85:673-5.

9 Eliassi-Rad B, Albert DM, Green WR. Frequency of ocular metastases in patients dying of cancer in eye bank populations. Br f Ophthalmol 1996;80:125-8.

10 Güthert H, Jänisch W, Rossbach K. Über die Häufigkeit der Augenmetastasen. Münch Med Wochenschr 1965;19:939Augen.

11 Nelson CC, Hertzberg BS, Klintworth GK. A histopatholic study of 716 unselected eyes in patients with cancer at the time of death. Am f Ophthalmol 1983;95:788-93.

12 Mewis L, Young SE. Breast carcinoma metastatic to the choroid-analysis of 67 patients. Ophthalmology 1982;89: $147-51$

13 Fleiss JC. Statistical methods for rates and proportions. New-York: Wiley, 1973.

$14 \mathrm{Chu}$ FCH, Huh SH, Nisce LZ, et al. Radiation therapy of choroid metastasis from breast cancer. Int $\mathcal{F}$ Radiat Oncol Biol Phys 1977;2:273-9.

15 Maor M, Chan CC, Young SE. Radiotherapy of choroidal metastases-breast cancer as primary site. Cancer 1977;40: 2081-6.

16 Minatel E, Trovo MG, Forner L, et al. The efficacy of radiotherapy in the treatment of intraocular metastases. $\mathrm{Br} \mathcal{F}$ Ophthalmol 1993;66:699-702.

17 Röttinger EM, Heckemann R, Scherer E, et al. Radiation therapy of choroidal metastases from breast cancer. Graefes Arch Klin Exp Ophthal 1976;200:243-50.

18 Rudoler SB, Shields CL, Corn BW, et al. Functional vision is improved in the majority of patients treated with external is improved in the majority of patients treated with external analysis of 188 patients. Int $\mathcal{F}$ Radiat Oncol Biol Phys 1996; 36 (Suppl 1):200.

19 Ratanatharathorn V, Powers WE, Grimm J, et al. Eye metastasis from carcinoma of the breast: diagnosis, radiation treatment and results. Cancer Treat Rev 1991;18:261-76. 